\section{(1) \\ CrossMark}

\title{
The inhaled corticosteroid/long-acting $\beta$-agonist maintenance and reliever therapy regimen: where to from here?
}

\author{
Richard Beasley $\mathbb{1}^{1}$, Irene Braithwaite $\mathbb{1}^{1}$, James Fingleton $\mathbb{1}^{1}$ and \\ Mark Weatherall (1) ${ }^{2}$
}

Affiliations: ${ }^{1}$ Medical Research Institute of New Zealand, Wellington, New Zealand. ${ }^{2}$ University of Otago, Wellington, New Zealand.

Correspondence: R. Beasley, Medical Research Institute of New Zealand, Private Bag 7902, Wellington 6242, New Zealand. E-mail: Richard.Beasleylamrinz.ac.nz

\section{@ERSpublications}

ICS/LABA maintenance and reliever therapy is recommended for adolescents with asthma at risk of severe exacerbations http://ow.ly/xhhO30gKZ6W

Cite this article as: Beasley R, Braithwaite I, Fingleton J, et al. The inhaled corticosteroid/long-acting $\beta$-agonist maintenance and reliever therapy regimen: where to from here? Eur Respir J 2018; 51: 1702338 [https://doi.org/10.1183/13993003.02338-2017].

Combination inhaled corticosteroid (ICS)/long-acting $\beta$-agonist (LABA) inhaler therapy (ICS/LABA therapy) represents the mainstay of the management of moderate and severe asthma, recommended in the Global Initiative for Asthma (GINA) guidelines for steps 3, 4 and 5 [1]. Indeed, in some countries, such as Australia, ICS/LABA therapy now represents the default ICS treatment, with a recent survey reporting that just over four out of every five adults with asthma who are prescribed ICS receive ICS/LABA therapy [2, 3]. As a result, the optimal use of ICS/LABA therapy is a priority in the management of asthma and this requires the consideration of a number of key issues. First, ICS and LABA should not be prescribed as separate inhalers in asthma due to the risks associated with LABA monotherapy in patients who are non-adherent with their ICS inhaler [4]. This recommendation reflects concern that LABA monotherapy increases the risk of mortality in patients with poorly controlled asthma, in contrast to ICS/LABA treatment for which no such risk has been identified [5]. Secondly, there are two main management regimens by which ICS/LABA inhalers can be prescribed. The most common "standard" regimen is fixed-dose ICS/LABA maintenance with a short-acting $\beta$-agonist (SABA) as reliever. The alternative option is to use a combination ICS/LABA inhaler as both maintenance and reliever therapy (referred to as the MART regimen). To implement the MART regimen it is necessary for the LABA component to have a fast onset of action, which allows for its use as a reliever as well as a maintenance therapy. The efficacy of the MART regimen has been illustrated with budesonide/formoterol using both turbuhaler [6] and metered dose inhaler (MDI) [7] devices for delivery, as well as with beclometasone/formoterol using the MDI device [8]. Thirdly, there is evidence that turbuhaler administration of budesonide/formoterol according to the MART regimen is more effective than the regimen comprising fixed-dose ICS/LABA maintenance (at a higher maintenance ICS dose) with "as required" SABA therapy in adults and children with chronic asthma [9]. In the Cochrane systematic review and meta-analysis summarising this evidence,

Received: Nov 132017 | Accepted: Nov 202017

Support statement: The Medical Research Institute of New Zealand is supported by Health Research Council of New Zealand Independent Research Organisation funding. Funding information for this article has been deposited with the Crossref Funder Registry.

Conflict of interest: Disclosures can be found alongside this article at erj.ersjournals.com

Copyright @ERS 2018 
the point estimates for the magnitude of the greater efficacy of the MART regimen were large. For the MART regimen versus the higher fixed-dose ICS/LABA regimen, the odds ratio for risk of exacerbations requiring hospitalisation or a visit to the emergency room was 0.72 (95\% CI $0.57-0.90$ ) while the odds ratio for exacerbations requiring oral corticosteroids was 0.75 (95\% CI $0.65-0.87$ ). This knowledge-base has now been extended by the publication, by JORup et al. [10] in this issue of the European Respiratory Journal, of an individual patient data subgroup meta-analysis which reports on the efficacy of the MART regimen in children aged 12-17 years. This meta-analysis includes 1847 adolescents from six double-blind randomised controlled trials comparing MART with an active comparator. Five trials required patients to have had at least one asthma exacerbation in the previous year at randomisation, thereby ensuring that the findings could be generalised to high risk adolescents with asthma. Comparator treatments used a SABA or LABA as the reliever therapy and included maintenance ICS at a dose of at least double the equivalent daily maintenance dose of ICS used in the MART regimen, or maintenance ICS/LABA at the same or a higher equivalent daily maintenance ICS dose, thereby representing the main treatment options from GINA steps 2, 3, 4 and 5 [1].

Overall, budesonide/formoterol self-administered according to the MART regimen reduced the risk of a first severe exacerbation, with a hazard ratio of 0.49 (95\% CI 0.34-0.70). As expected in an analysis involving small numbers of adolescents in each study, with different comparator treatments, there was significant heterogeneity. Budesonide/formoterol MART was more effective than fixed-dose ICS regimens and was comparable to, or more effective than, fixed-dose ICS/LABA regimens. In contrast to the exacerbation findings, the benefit in terms of asthma control was not clinically significant (the mean difference in the Asthma Control Questionnaire (ACQ) was - $0.07,95 \%$ CI -0.15-0.01). While the authors suggest that the MART regimen in adolescents has comparable efficacy to that in adults, noting that the hazard ratio for time to first severe exacerbation in adults was 0.65 (95\% CI $0.50-0.72$ ), the reporting of interaction tests comparing adults with adolescents would have been informative and would have enabled us to better understand the strength of evidence supporting this interpretation.

These findings are of major clinical significance as children, including adolescents, are a high risk group with a substantive burden from asthma, including the risk of severe exacerbations [11]. Key factors contributing to the risk of severe exacerbations in adolescents are poor adherence to maintenance ICS therapy and poor adherence to oral corticosteroids after emergency room visits or hospital admissions, both of which are related to independent and/or risk-taking behaviours, anxiety and depression [12-14]. The capability of the MART regimen to reduce the number of days in which no ICS is self-administered and the number of days of $\beta$-agonist overuse associated with a delay in obtaining medical help, both of which have been demonstrated in adult asthma [7], is also likely to apply to adolescents and contribute to its efficacy in this patient group.

So where to from here? It will be necessary for evidence-based national and international asthma guidelines to make recommendations that the MART regimen is the preferred ICS/LABA regimen in both adolescents and adults at risk of severe exacerbations. In the interim, while guidelines are updated, clinicians who currently preferentially prescribe ICS/LABA regimens according to fixed-dose once or twice daily protocols will need to consider changing their practice in accordance with currently available evidence and preferentially prescribe the MART regimen for those with "high risk" asthma. However, this is a complex issue as there are many factors in addition to scientific evidence that determine prescribing, including regulatory approval, pharmaceutical cost and funding, doctor and patient knowledge, and preference (including familiarity with different inhaler devices). It will be important that widespread regulatory approval is obtained for the prescription of budesonide/formoterol according to the MART regimen with MDI devices, as well as with the turbuhaler as currently available, and that beclometasone/ formoterol MDI devices are more widely available globally.

What about the future? The observation that ICS/fast-onset LABA reliever therapy is more effective than SABA reliever therapy in patients receiving maintenance ICS/LABA therapy $[6,9]$ raises the question as to whether the former may likewise be more effective than the latter in patients with intermittent or mild asthma who are not prescribed any regular maintenance ICS or ICS/LABA therapy. It also raises the question as to whether an ICS/fast-onset LABA reliever regimen might be more effective than a fixed-dose maintenance ICS and SABA reliever regimen in patients with mild asthma, in whom ICS adherence is an inherent difficulty, as it would allow titration of ICS according to changes in asthma symptom control. The results of the current clinical trial programme investigating these questions are keenly awaited [15-17]. A potential alternative is to use a combination ICS/SABA inhaler as a reliever therapy, instead of a SABA reliever therapy, in intermittent and mild asthma. In this regard there is emerging evidence that ICS/SABA reliever therapy is superior to SABA reliever therapy, and non-inferior to regular maintenance ICS and SABA reliever therapy, in both children and adults with mild asthma [18]. Further studies of ICS/SABA reliever therapy across the broad range of asthma severity are a high priority in order to determine 
whether a combination ICS/salbutamol inhaler may represent a bronchodilator with a better efficacy/safety profile than salbutamol reliever therapy, as suggested in a recent review [19].

\section{References}

1 Global Initiative for Asthma. Global Strategy for Asthma Management and Prevention, 2017. http://ginasthma.org/ 2017-gina-report-global-strategy-for-asthma-management-and-prevention/ Date last accessed: November 22, 2017.

2 Beasley R, Hardy J, Hancox R. Asthma prescribing: where are we headed? Respirology 2017; 22: 1487-1488.

3 Reddel HK, Beckert L, Moran A, et al. Is higher population-level use of ICS/LABA combination associated with better asthma outcomes? Cross-sectional surveys of nationally representative populations in New Zealand and Australia. Respirology 2017; 22: 1570-1578.

4 Beasley R, Perrin K, Weatherall M, et al. Call for withdrawal of LABA single inhaler therapy in asthma. Lancet 2010; 376: 750-751.

5 Weatherall $\mathrm{M}$, Wijesinghe $\mathrm{M}$, Perrin $\mathrm{K}$, et al. Meta-analysis of the risk of mortality with salmeterol and the effect of concomitant inhaled corticosteroid therapy. Thorax 2010; 65: 39-43.

6 Rabe KF, Atienza T, Magyar P, et al. Effect of budesonide in combination with formoterol for reliever therapy in asthma exacerbations: a randomised controlled, double-blind study. Lancet 2006; 368: 744-753.

7 Patel M, Pilcher J, Pritchard A, et al. Efficacy and safety of maintenance and reliever combination budesonide-formoterol inhaler in patients with asthma at risk of severe exacerbations: a randomised controlled trial. Lancet Respir Med 2013; 1: 32-42.

8 Papi A, Corradi M, Pigeon-Francisco C, et al. Beclometasone-formoterol as maintenance and reliever treatment in patients with asthma: a double-blind, randomised controlled trial. Lancet Respir Med 2013; 1: 23-31.

9 Kew KM, Karner C, Mindus SM, et al. Combination formoterol and budesonide as maintenance and reliever therapy versus combination inhaler maintenance for chronic asthma in adults and children. Cochrane Database Syst Rev 2013; CD009019.

10 Jorup C, Lythgoe D, Bisgaard $\mathrm{H}$. Budesonide/formoterol maintenance and reliever therapy in adolescent patients with asthma. Eur Respir J 2018; 51: 1701688.

11 Rabe KF, Vermeire PA, Soriano JB, et al. Clinical management of asthma in 1999: the Asthma Insights and Reality in Europe (AIRE) study. Eur Respir J 2000; 16: 802-807.

12 Desai M, Oppenheimer JJ. Medication adherence in the asthmatic child and adolescent. Curr Allergy Asthma Rep 2011; 11: 454-464.

13 Bender BG. Risk taking, depression, adherence, and symptom control in adolescents and young adults with asthma. Am J Respir Crit Care Med 2006; 173: 953-957.

14 Bitsko MJ, Everhart RS, Rubin BK. The adolescent with asthma. Paediatr Respir Rev 2014; 15: 146-153.

15 O'Byrne PM, FitzGerald JM, Zhong N, et al. The SYGMA programme of phase 3 trials to evaluate the efficacy and safety of budesonide/formoterol given 'as needed' in mild asthma: study protocols for two randomised controlled trials. Trials 2017; 18: 12.

16 Beasley R, Pavord I, Papi A, et al. Description of a randomised controlled trial of inhaled corticosteroid/fast-onset LABA reliever therapy in mild asthma. Eur Respir J 2016; 47: 981-984.

17 Fingleton J, Hardy J, Baggott C, et al. Description of the protocol for the PRACTICAL study: a randomised controlled trial of the efficacy and safety of ICS/LABA reliever therapy in asthma. BMJ Open Respir Res 2017; 4: e000217.

18 Beasley R, Weatherall $\mathrm{M}$, Shirtcliffe $\mathrm{P}$, et al. Combination corticosteroid/ $\beta$-agonist inhaler as reliever therapy: a solution for intermittent and mild asthma? J Allergy Clin Immunol 2014; 133: 39-41.

19 O'Byrne PM, Jenkins C, Bateman ED. The paradoxes of asthma management: time for a new approach? Eur Respir J 2017; 50: 1701103. 